\title{
LIFE-THREATENING HYPERKALEMIA PRESENTING WITH CARDIAC ARREST DURING COMBINED THERAPY WITH ANGIOTENSIN - CONVERTING ENZYME INHIBITOR AND SPIRONOLACTONE: A CASE REPORT
}

\author{
CHAKRABORTY $\mathrm{S}^{1}$, KHAYER MA², RAHMAN M ${ }^{1}$
}

\begin{abstract}
Abstarct
A 56-year-old man with history of previous coronary artery bypass graft (CABG) surgery and prior history of mild renal impairment was brought to the emergency department at mid night in gasping state. On presentation his pulse and blood pressure (BP) were non recordable. Cardiopulmonary resuscitation (CPR) was started immediately and he was intubated in the emergency room. Within few minutes his pulse became perceptible but BP still remained non recordable. His bed side 12 leads electrocardiogram (ECG) revealed sine wave configuration, suggestive of severe hyperkalemia with heart rate 30-35 beats per minute (bpm).Urgently he was transferred to cardiac catheterization laboratory for temporary pacemaker insertion (TPI). After putting TPI he regained consciousness and BP raised to normal. His blood gas analysis showed severe hyperkalemia (Serum potassium level-9.4 $\mathrm{mmol} / \mathrm{L}$ ). In coronary care unit (CCU) he was commenced on standard treatment of hyperkalemia and his electrolyte imbalance normalized within 24 hours. His cardiac rhythm reverted back to sinus within 24 hours of admission and he was extubated next day and discharged from the hospital after 5 days in a relatively stable state.
\end{abstract}

Key words: Hyperkalemia, Cardiac arrest, Sine wave.

J Dhaka Med Coll. 2019; 28(2) : 212-215

\section{Introduction}

Hyperkalemia is defined as a serum potassium $\left(\mathrm{K}^{+}\right)$concentration greater than approximately $5.0-5.5 \mathrm{mmol} / \mathrm{L}$ in adults. Levels higher than 7 $\mathrm{mmol} / \mathrm{L}$ can lead to significant hemodynamic and neurologic consequences, whereas levels exceeding $9.0 \mathrm{mmol} / \mathrm{L}$ can cause respiratory paralysis or cardiac arrest and can quickly be fatal. ${ }^{1-2}$ The most common clinical presentation of severe hyperkalemia involves patients with end-stage renal failure. These patients may present with severe weakness or arrhythmias. ${ }^{3-}$

4 Medications may also contribute to development of hyperkalemia, particularly in the presence of impaired renal function. Potassium-sparing diuretics such as spironolactone, triamterene, and amiloride are well-recognized causes of hyperkalemia. Use of angiotensin-converting enzyme (ACE) inhibitors (eg,ramipril) and angiotensin II receptor blockers (ARBs) (eg.losartan) can also lead to marked elevation of serum potassium, particularly when combined with potassiumsparing diuretics. ${ }^{5}$ Identification of potential causes of hyperkalemia will contribute to rapid identification and treatment of patients who

1. Dr. Shudipan Chakraborty, Resident Medical Officer, Labaid Cardiac Hospital, Dhaka.

2. Dr. M A Khayer, Consultant Cardiologist, Labaid Cardiac Hospital,,Dhaka.

3. Dr.Mahbubor Rahman, Senior Consultant Cardiologist and In-Charge Coronary Care Unit, Labaid Cardiac Hospital, Dhaka.

Correspondence: Dr. Shudipan Chakraborty, Resident Medical Officer, Labaid Cardiac Hospital, Dhanmondi, Dhaka 1205. Mobile 01923626609, E-mail: shuchak91@gmail.com 
may be experiencing hyperkalemic cardiac arrhythmias and cardiac arrest.

\section{Case Report}

We report a case of a 56-year-old man with history of previous CABG surgery and mild renal impairment who suddenly collapsed in his residence and was brought to the emergency department at mid night in gasping state. As he was unconscious and pulse and BP were non recordable, he was intubated immediately and CPR was started in the emergency room. Within 15 minutes of starting CPR his pulse became perceptible but $B P$ still remained non recordable. His bed side ECG revealed sine wave configuration, suggestive of severe hyperkalemia with heart rate $30-35$ bpm (Fig1). Urgently he was transferred to cardiac catheterization laboratory for temporary pacemaker insertion (TPI). After putting TPI he regained consciousness and $\mathrm{BP}$ became recordable. Eventually he was transferred to coronary care unit (CCU) for further management. In CCU his systemic examination revealed normal pulse rate (on TPI) with normal BP but auscultation of lungs revealed bilateral coarse crepitations. After admission in CCU on query his relatives admitted that the patient was not well for the last few days. At home he was anorexic, lethargic with mild shortness of breath and had several episodes of vomiting before he collapsed.

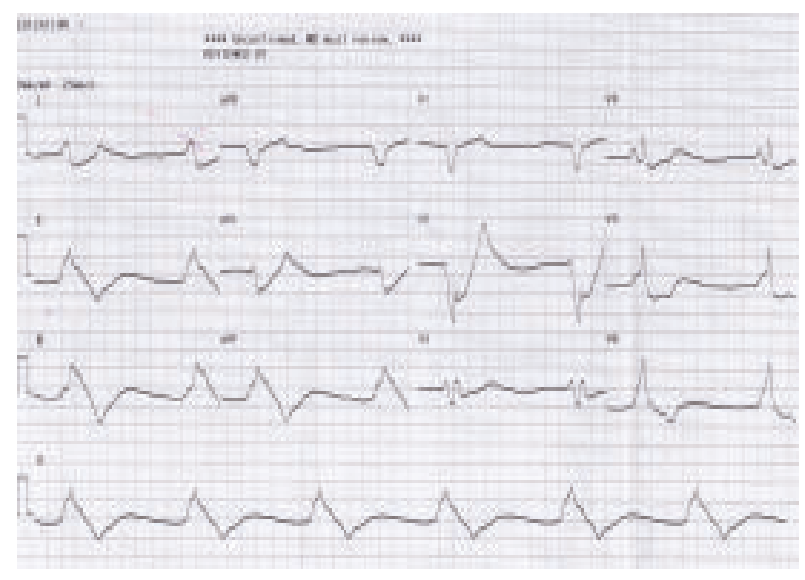

Fig-1. ECG shows wide and bizarre QRS complex with sine wave configuration with marked bradyarrhythmia suggestive of severe hyperkalemia during admission (Serum potassium level $-9.4 \mathrm{mmol} / \mathrm{L}$ ).
As history dates back, this patient had acute coronary syndrome (ACS), 5 years back and his coronary angiogram was suggestive triple vessel disease. Eventually he underwent CABG operation. But after operation, his left ventricular ejection fraction (LVEF) was around $35 \%$. During past two years he was admitted twice in hospital with symptoms of left ventricular failure (LVF). He was non-smoker, hypertensive for 10 years, diabetic for 15 years and was on insulin, and had mild renal impairment with serum creatinine level ranging from 1.5-2.0 mg/dL. He was regularly on tab.ramace (ramipril) $2.5 \mathrm{mg}$ twice daily, tab. edeloss Plus (furosemide $40 \mathrm{mg}+$ spironolactone $50 \mathrm{mg}$ ) once daily in the morning along with other cardiac medicines.

In CCU his blood gas analysis showed severe hyperkalemia (serum potassium level-9.4 $\mathrm{mmol} / \mathrm{L}$ ) and treated as such. Treatment of the patient's hyperkalemia with glucose-insulin, intravenous furosemide, nebulization with Bagonist, calcium, $\mathrm{K}^{+}$exchange resin, kayexalate, were initiated without any delay. On the day of admission his serum creatinine level was 2.5 $\mathrm{mg} / \mathrm{dL}$. After commencement of standard treatment his clinical condition gradually improved and his check potassium level 4 hours after admission was noted to be $7.3 \mathrm{mmol} / \mathrm{L}$ and same treatment was continued. The patient regained consciousness within 6 hours of admission. On the following day as his cardiac rhythm reverted back to sinus, temporary pacemaker was withdrawn and he was extubated on the same day. On second day

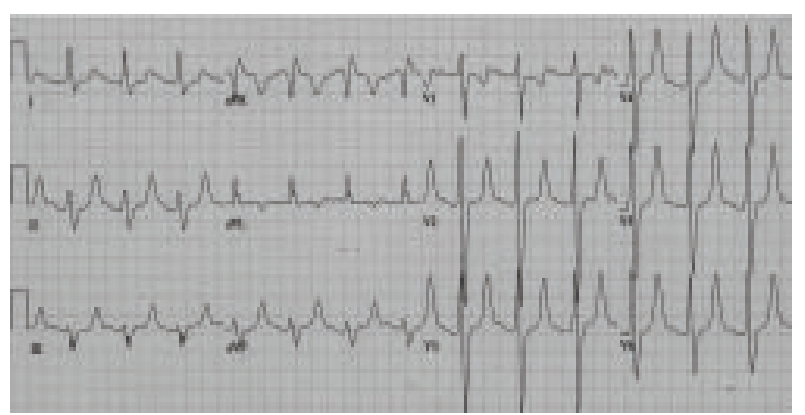

Fig-2. ECG of the same on second day of admission showing features of hyperkalemia with tall peaked $T$ waves, flattened $P$ waves, prolonged $P R$ interval and widened $Q R S$ complex (Serum potassium level - $6.6 \mathrm{mmol} / \mathrm{L}$ ). 
morning his $\mathrm{K}^{+}$level was noted to be $6.6 \mathrm{mmol} /$ $\mathrm{L}$ and ECG still revealed some features of hyperkalemia with tall peaked $\mathrm{T}$ waves, flattened $\mathrm{P}$ waves,prolonged $\mathrm{PR}$ interval and widened QRS complex (Fig-2).His further hospital course was uneventful and his serum $\mathrm{K}^{+}$level was nomalized within 48 hours of admission with continuing conservative treatment and he was discharged from the hospital on $5^{\text {th }}$ day of admission in a reasonably stable state.

\section{Discussion}

Hyperkalemic cardiac arrest is a severe but not uncommon complication of hyperkalemia. As potassium plays a critical role in cellular depolarization and tissue excitability, minor fluctuations in extracellular levels can have significant pathologic effects, potentially leading to cardiac dysrhythmias and cardiac arrest. ${ }^{3}$ Hyperkalemia is most commonly caused by renal failure or due to medication side effects; however, many cases are multifactorial. The case we presented here, hyperkalemia was induced by combination of pre existing renal impairment and simultaneous use of ACEI (ramipril) and potassium-sparing diuretic (spironolactone) combination. It is important to be able to recognize hyperkalemia and to be familiar with its treatment as hyperkalemia affects as many as $8 \%$ of hospitalized patients. ${ }^{4-5}$ The pattern of ECG findings with hyperkalemia depends on serum $\mathrm{K}^{+}$levels, with initial ECG changes being noted at serum concentrations between 5.5 and $6.5 \mathrm{mmol} / \mathrm{L}$ and serious, potentially fatal arrhythmias not being seen until serum $\mathrm{K}^{+}$concentration approach $9.0 \mathrm{mmol} / \mathrm{L}$.ECG changes suggestive of hyperkalemia include tall and peaked $T$ waves (tenting), flattened $\mathrm{P}$ waves, prolonged PR interval (first-degree heart block), widened QRS complex, deepened $\mathrm{S}$ waves and merging of $\mathrm{S}$ and $\mathrm{T}$ waves,idioventricular rhythm, sine-wave formation, ventricular fibrillation (VF) and cardiac arrest.Tenting of $\mathrm{T}$ waves is one of the prominent early ECG changes. If untreated, hyperkalemia causes progressive heart dysfunction, leading to sine waves and finally to asystole. ${ }^{5,6}$ Aggressive therapy should begin as soon as possible to improve outcome. Our case presented in the emergency department in a state of cardiac arrest and after CPR, bedside ECG revealed sine wave configuration, which is harbinger of death if not properly treated. In our case as the patient was having severe bradycardia and non recordable BP, temporary pacemaker was inserted and patient became hemodynamically stable and regained consciousness. TPI may be a life-saving procedure in patients with severe bradyarrhythmia and cardaic arrest. ${ }^{7,8}$ In our patient cardiac rhythm was restored immediately after TPI and he recovered without any neurological sequelae.

Intravenous (IV) calcium, insulin with dextrose infusion, IV sodium bicarbonate, nebulized salbutamol and cation exchange resins are the standard treatment of hyperkalemia. Our patient was commenced on IV calcium gluconate and IV glucose/insulin immediately after admission. Resistant hyperkalemia may require emergency dialysis. Temporary pacing is a life-saving procedure with or without dialysis. ${ }^{9,10}$ In this particular patient, cardaic rhythm was restored by TPI and hyperkalemia was quickly normalized by medication with repeated calcium gluconate and glucose/insulin infusion and cation exchange resin.

\section{Conclusion:}

Severe hyperkalemia is a potential lifethreatening cardiac emergency especially in the patients with renal impairment who are on combination ACEI and potassium-sparing diuretics. Various conventional therapies including intravenous furosemide, insulin with glucose, IV calcium and several beta-2 agonists are commonly employed as transient measures to enhance shift of potassium from the extracellular to the intracellular compartment. In presense of severe bradyarrhythmia along with haemodynamic instability temporary pacing may prevent unfortunate fatal outcome. Hyperkalemia is a commonly encountered electrolyte abnormality that can lead to lifethreatening derangements in cardiac conduction leading to cardiac arrest. The emergency medicine physician and specialist should be aware of the range of dysrhythmia attributed to hyperkalemia. 


\section{References:}

1. Ingelfinger J. A new era for the treatment of hyperkalemia. N Engl J Med. 2015; 372:275-277.

2. Sterns RH, Grieff M, Bernstein PL. Treatment of hyperkalemia: something old, something new. Kidney Int. 2016; 89:546-554.

4. Kiran S, Rohan S, Shelley Z.Hyperkalemia in heart failure. Curr Opin Cardiol. 2020; 35(2): 150-155

4. Beusekamp JC, Tromp J, Cleland JGF, et al. Hyperkalemia and treatment with RAASinhibitors during acute heart failure hospitalizations and their association with mortality. JACC Heart Fail. 2019; 7:970-979.

5. Bakris GL, Siomos M, Richardson D, et al. ACE inhibition or angiotensin receptor blockade: impact on potassium in renal failure. Kidney Int. 2000; 58:2084-2092.
6. Varga C,Kalman Z,Szakail A et al.ECG alterations suggestive of hyperkalemia in normokalemic versus hyperkalemic patients.BMC Emerg Med.2019;19:33-38.

7. Dwivedi S K, Kumar P, Chaudhary G et al.Lifethreatening Hyperkalemia: Can aggressive medical therapy defer temporary pacing. Heart India. 2014; 2:59-61.

8. Oh P C,Koh K K,Kim J H et al.Life threatening severe hyperkalemia presenting typical electrocardiographic changes-Rapid recovery following medical, temporary pacing, and hemodialysis treatments. Int J Cardiol. 2014;177:27-29.

9. Kosovali BD, Yildiz H. Reversible complete atrioventriculer block in patient with mild hyperkalemia. J Cardiol Curr Res. 2018;11(1): 18-19.

10. Agarwal N, Singh A, Gaba R, et al. Transient trifascicular block in severe hyperkalemia: A case report. J Assoc Physicians India 2015;63(9): 74-75. 